
\title{
R Research S Suare \\ Infection and transmission of the facultative endosymbiont Arsenophonus in the spider Pardosa pseudoannulata
}

Hanqi Jiang

Nanjing Agricultural University

Yu Ding

Chinese Academy of Agricultural Sciences

Dongxiao Zhao

Jiangsu Academy of Agricultural Sciences

Xiangdong Liu

Nanjing Agricultural University

Huifang Guo ( $\nabla$ guohfjaas@163.com )

Jiangsu Academy of Agricultural Sciences

Research article

Keywords: Pardosa pseudoannulata, Arsenophonus, geographic population, vertical transmission

Posted Date: September 8th, 2020

DOI: https://doi.org/10.21203/rs.3.rs-48416/v1

License: (9) This work is licensed under a Creative Commons Attribution 4.0 International License.

Read Full License 


\section{Abstract}

Background The infection of insect pests and their parasitoids with the facultative endosymbiont Arsenophonus has been well studied, whereas the infection of the predators of insect pests with this symbiont is less well understood. We studied this symbiont in the spider Pardosa pseudoannulata, an important predator of rice planthoppers, collected from rice paddies in 8 geographical regions in China.

Results Diagnostic PCR showed that Arsenophonus was present in 4 of the 8 sampled populations of spiders. In these 4 populations, the infection incidence was between $2.1 \%$ and $16.7 \%$ and significantly differed between the Lishui population and the other three populations. Investigation of symbiont infection in both females and their offspring indicated that Arsenophonus can be vertically transmitted in P. pseudoannulata; the average transmission rate was approximately $25 \%$. Remarkably, Arsenophonus was present in approximately $11 \%$ of the offspring of uninfected females, indicating that nonmaternal transmission occurs in this spider.

Conclusions Populations of the spider P. pseudoannulata are infected at low rates with the symbiont Arsenophonus. This symbiont can be vertically transmitted from female spiders to offspring but may also be transmitted by male spiders or other vectors. Arsenophonus populations from different geographical regions exhibit genetic differentiation.

\section{Background}

Endosymbiotic bacteria in arthropods have received considerable attention due to their multiple roles in host ecology and evolution (Nyffeler \& Benz, 1987; Rowley et al., 2004; Pontes \& Dale, 2006; Duron et al., 2008; Zug \& Hammerstein, 2015; Liu \& Guo, 2019). At least 10 species of symbionts, belonging to the genera Wolbachia, Arsenophonus, Cardinium, Rickettsia, Spiroplasma and Rickettsiella, have been reported in arthropods (Nyffeler \& Benz, 1987; Rowley et al., 2004; Goodacre et al., 2006; Duron et al., 2008; Vanthournout et al., 2011; Stefanini \& Duron, 2012; Zug \& Hammerstein, 2015; Zhang et al., 2018; Liu \& Guo, 2019). Among these symbiont taxa, Wolbachia is the most common and has been found in many species of insect pests (Zug \& Hammerstein, 2015, Liu \& Guo, 2019). It is also present in spiders, which are important predators of insect pests in terrestrial ecosystems (Rowley et al., 2004; Goodacre et al., 2006; Vanthournout et al., 2011; Stefanini \& Duron, 2012). Similar to Wolbachia, the genus Arsenophonus is a clade of symbiotic bacteria with a vast host distribution. The known host spectrum of Arsenophonus encompasses diverse insect groups, including parasitic wasps, triatomine bugs, psyllids, whiteflies, aphids, ticks, ant lions, hippoboscids, streblids, bees, lice and others, and noninsect taxa, including two plant species (Gherna et al., 1991; Chiel et al., 2007; Hansen et al., 2007; Nováková et al., 2009; Wagner et al., 2015). Arsenophonus can be vertically and horizontally transferred in parasitoids (Gherna et al., 1991; Huger et al., 1985). For example, the horizontal transfer of Arsenophonus infection in Nasonia wasps occurs after multiparasitism events in which two species of parasitoids share a pupal host (Huger et al., 1985; Duron et al., 2010). 
However, whether Arsenophonus infection and transmission occur in spiders is unknown. Does Arsenophonus infection occur in spiders? If so, does the infection vary among geographical populations of the host, and how does transmission occur? In this study, we attempt to answer the above questions by focusing on the spider Pardosa pseudoannulata, which is an important predator in rice paddies (Liu et al., 1999). We compared Arsenophonus infections among different geographical populations of $P$. pseudoannulata from rice paddies in China and investigated the transmission of Arsenophonus in this spider.

\section{Methods}

\section{Spider collection}

pseudoannulata individuals were collected from 8 sites of rice paddies in China between August 2015 and October 2018 (Table 1). At each site, each individual was collected at least $30 \mathrm{~m}$ from other collected individuals, and the spiders were collected from at least five rice paddies at each site. Spiders without egg bags were chosen for direct symbiont detection. Spiders carrying egg bags were chosen for the investigation of symbiont transmission. The spiders were fed on small brown planthoppers uninfected with any known bacterial symbionts (the symbiont-free planthoppers were the same as those previously described (Li et al., 2018) and were reared separately in laboratory conditions at $27 \pm 1^{\circ} \mathrm{C}$ with a $14: 10 \mathrm{~h}$ (L:D) photoperiod.

Table 1 Collection information of Pardosa pseudoannulata individuals without egg bags

\begin{tabular}{|lllll|}
\hline Collection site (code) & No. of samples & Longitude & Latitude & Collection time \\
\hline Nan Jing, Jiangsu (NJ) & $6^{*}$ & $118^{\circ} 86^{\prime} \mathrm{E}$ & $32^{\circ} 03^{\prime} \mathrm{N}$ & Aug. 2015 \\
\hline LiShui, Jiangsu (LS) & 18 & $118^{\circ} 86^{\prime} \mathrm{E}$ & $32^{\circ} 03^{\prime} \mathrm{N}$ & June 2018 \\
\hline YangZhou, Jiangsu (YZ) & 11 & $119^{\circ} 18^{\prime} \mathrm{E}$ & $31^{\circ} 60^{\prime} \mathrm{N}$ & Oct. 2018 \\
\hline JuRong, Jiangsu (JR) & $96,5^{\star}$ & $119^{\circ} 25^{\prime} \mathrm{E}$ & $32^{\circ} 21^{\prime} \mathrm{N}$ & Feb. 2018 \\
\hline FengYang, Anhui (FY) & 7 & $119^{\circ} 25^{\prime} \mathrm{E}$ & $32^{\circ} 00^{\prime} \mathrm{N}$ & Aug. 2015 \\
\hline NanCang, Jiangxi (NJ) & 13 & $117^{\circ} 56^{\prime} \mathrm{E}$ & $32^{\circ} 87^{\prime} \mathrm{N}$ & Feb. 2018 \\
\hline HenYang, Hunan (HY) & 22 & $115^{\circ} 49^{\prime} \mathrm{E}$ & $28^{\circ} 55^{\prime} \mathrm{N}$ & June 2017 \\
\hline ChuXiong, Yunnan (CX) & 7 & $112^{\circ} 57^{\prime} \mathrm{E}$ & $26^{\circ} 89^{\prime} \mathrm{N}$ & June 2018 \\
\hline
\end{tabular}

Numbers followed by ' $*$ ' are the numbers of adult spiders with egg bags.

\section{Detection of Arsenophonus infection in P. pseudoannulata}


To compare Arsenophonus infection among different geographic populations of $P$. pseudoannulata, the presence of the symbiont in spiders without egg bags collected from different sites was evaluated by diagnostic PCR using specific primers (23SF 5'CGTTTGATGAATTCATAGTCAAA3', 23SR

5 'GGTCCTCCAGTTAGTGTTACCCAAC3') (Thao et al., 2000). To rule out the possibility of detecting the bacteria from spider gut, we only used spider legs to extract DNA to detect the presence of Arsenophonus. DNA from the legs of each P. pseudoannulata was extracted using the Wizard $\circledast$ Genomic DNA Purification Kit (Promega). DNA quality was examined using a spectrophotometer. Only DNA samples with an OD260/OD280 ratio ranging from 1.6 to 1.9 were used for PCR to detect symbiont species. Both positive and negative controls were used. The presence of Arsenophonus was assessed following previously described methods (Thao et al., 2000). To verify the presence of the symbionts and perform comparisons among hosts, the PCR products were isolated and sent to Sangon Biotech Co. (Shanghai, China) for sequencing. The sequences were deposited in the National Center for Biotechnology Information (NCBI) under accession numbers MN368165, MN368167 and MN368167.

\section{Transmission of Arsenophonus in P. pseudoannulata}

To study the transmission of Arsenophonus in P. pseudoannulata, spiders with egg bags were used to establish isofemale lines. All the adults and offspring were reared separately, and both adults and offsprings were used to detect symbiont infection.

After the spider eggs hatched, the adult females were used to detect the presence of all known six symbionts found in rice planthoppers and spiders, including Arsenophonus, Acinetobacter, Wolbachia, Serratia, Cardinium and Spiroplasma; the methods used were as previously described for Arsenophonus (Thao et al., 2000), Cardinium (Nakamura et al., 2009), Acinetobacter(Vanbroekhoven et al., 2004), Wolbachia (Zhou et al., 1998), Serratia (Zhu et al., 2008) and Spiroplasma (Sanada-Morimura et al., 2013). The whole body of each offspring was used to identify Arsenophonus infection in each individual. The transmission rate of each isofemale was calculated according to the infected number and the total number of offspring.

\section{Statistics}

To explore the genetic relationships among the Arsenophonus sequences detected in different spider populations, phylogenies were constructed using maximum-likelihood and neighbor-joining methods. The sequences were imported and aligned using MEGA7, and genetic distance was estimated with the Kimura 2-parameter model. The $23 \mathrm{~S}$ rRNA sequences of Arsenophonus in other species (Dialeurodes hongkongensis AY264667.1, Aphis glycines KC019882.1, Neomaskellia andropogonis AY264668.1, Tetraleurodes acaciae AY264670.1, Ericerus pela JN990930.1, Acanthaleyrodes styraci AY264663.1, Nilaparvata lugens KX280767.1, Bemisia tabaci AB981361.1, Aleurodicus dugesii AY587142.1, and Chilo suppressalis JF719284.1) were used as outgroups.

Differences in the infection incidence of Arsenophonus among different geographic populations and differences in transmission rate among isofemales were evaluated using the Chi-square test. The 
infection incidence in offspring from both Arsenophonus-infected and noninfected mothers was analyzed by Student $t$ test. The statistical analyses were performed using SPSS 19.0 software.

\section{Results}

\section{Natural infection with Arsenophonus in different geographical populations of $P$. pseudoannulata}

The incidence of Arsenophonus infection in P. pseudoannulata collected in 8 sites from Jiangsu, Jiangxi, Anhui, Hunan and Yunnan Provinces, China, were compared among the spider populations.

Arsenophonus was found to be present at 4 out of the 8 sites, where the infection incidence ranged from $2.1 \%$ to $16.7 \%$ and was highest in the LS population. The incidence in the LS population was significantly higher than that in the $\mathrm{NJ}\left(c^{2}=5.639, d f=1, P=0.018\right), \mathrm{JR}\left(c^{2}=7.687, d f=1, P=0.006\right)$ and $\mathrm{HY}\left(c^{2}=3.964\right.$, $d f=1, P=0.046)$ populations. In contrast, none of the spiders collected in Yangzhou, Jiangsu Province; Fengyang, Anhui Province; Henyang, Hunan Province and Chuxiong, Yunnan Province were found to be infected with Arsenophonus. Across all 292 individuals collected from these 8 sites, the average incidence of infection with Arsenophonus was 3.4\% (Fig. 1).

\section{Coinfection of other symbionts with Arsenophonus in female $P$. pseudoannulata}

Five out of six known symbiont taxa in spiders and rice planthoppers, Wolbachia, Arsenophonus, Acinetobacter, Serratia, and Cardinium, were found in females of $P$. pseudoannulata carrying egg bags collected in Jurong and Nanjing, Jiangsu Province, China; Spiroplasma was not detected. In the 21 female spiders with egg bags, 13 patterns of symbiont infection were detected. Six out of 21 isofemales were found to be infected with Arsenophonus, and 4 infection patterns were observed, including single infection with Arsenophonus and coinfection with one, three or four symbionts (Cardinium, Serratia, Acinetobacter and Wolbachia). The other 15 females were not infected with Arsenophonus but were infected with one, two or three other symbionts. Among the 21 females, 17 (81.0\%), 6 (28.6\%), and 6 (28.6\%) females were infected with Serratia, Wolbachia, and Cardinium, respectively, and besides Arsenophonus, these symbionts were the dominant taxa in the spiders.

\section{Transmission of Arsenophonus}

The transmission of Arsenophonus between generations of $P$. pseudoannulata was examined in the 21 established isofemales. Six of the 21 isofemales were established from spiders with egg bags infected with Arsenophonus, and the remaining 15 were established from spider with egg bags lacking the symbiont. In the 6 isofemales from Arsenophonus-infected mothers, the symbiont was found in the next generation. The highest incidence of infection with Arsenophonus in the next generation was $46.7 \%$, and the lowest was $17.5 \%$. There were no significant differences in the incidence in offspring among these 6 isofemales $\left(\chi^{2}=7.477, d f=5, P=0.188\right)$. The average transmission rate was $25.03 \pm 4.78 \%$ in the Arsenophonus-infected mother spiders (Fig. 2A). 
In the 15 isofemales established from noninfected $P$. pseudoannulata females, the symbiont was found in the next generation of 12 isofemales. The highest incidence of infection with Arsenophonus in the next generation was $25 \%$, and the lowest was $5.8 \%$. No significant difference in infection incidence in offspring was found among the 12 isofemales $\left(\chi^{2}=11.577, d f=11, P=0.396\right)$. The infection rate of offspring from noninfected mothers was $11.11 \pm 2.13 \%$ (Fig. 2B), which was significantly lower than that of offspring of Arsenophonus-infected mothers $(t=3.108, d f=19, P=0.006)$.

\section{Genetic differentiation of Arsenophonus among different geographical populations of $P$. pseudoannulata}

Based on the 23S rRNA gene sequences of Arsenophonus, a close genetic relationship was found among the NC, LS and NJ spider populations from Jiangxi and Jiangsu Provinces (with a maximum distance of nearly $700 \mathrm{~km}$ among the 3 sites). The analysis revealed low genetic differentiation of Arsenophonus among these three geographic populations (Fig. 3).

Fig. 3 Phylogenetic tree based on the 23S rRNA gene sequences of Arsenophonus in Pardosa pseudoannulata and insect species using the neighbor-joining method. Numbers indicate bootstrap percentages at each node (1000 bootstraps). The nucleotide sequences of PCR-amplified fragments of Arsenophonus genes in our studies have been deposited in the GenBank nucleotide database under accession nos. MN368165 to MN368167.

\section{Discussion}

Previous extensive surveys have demonstrated that at least one-third of arthropod species are infected by a diverse range of vertically transmitted bacteria that strongly influence host biology (Huger et al., 1985; Liu \& Guo, 2019). The genus Arsenophonus is comprised of intracellular symbionts with a vast host distribution (Huger et al., 1985 Nováková et al., 2009). In rice paddies, the symbiont Arsenophonus has been found in many populations of insect pests, such as Nilaparvata lugens, at low infection rates (< 30\%) (Wang et al., 2012; Qu et al., 2013; Jiang et al., 2017). In this study, we found that Arsenophonus was present in the spider P. pseudoannulata, which is a main predator of rice planthoppers. We found that $62.5 \%$ (5 out of 8 ) of the tested spider populations were infected with Arsenophonus at low infection frequencies (with an average infection rate of $3.8 \%$ across the 8 sites, with a total of 292 spiders), which is similar to the infection level $(<30 \%)$ reported in rice planthoppers (Jiang et al., 2017). We also found that Arsenophonus could coinfect with other symbionts, including Cardinium, Serratia, Acinetobacter and Wolbachia, in P. pseudoannulata. Multi-infection has been found in the dwarf spider Oedothorax gibbosus, with coinfection with Wolbachia, Rickettsia and Cardinium detected (Vanthournout \& Hendrickx, 2015). A high diversity of Arsenophonus bacteria has been found in different insect species; in some cases, a single insect species carries multiple Arsenophonus strains. Fox example, Arsenophonus was found to be genetically differentiated in Bemisia tabaci (Thao et al., 2004; Mouton et al., 2012). However, in the spider P. pseudoannulata, no genetic differentiation of Arsenophonus was found among the different geographic populations of the spider. We only investigated genetic differences among three geographical population of $P$. pseudoannulata. Therefore, to confirm whether there is genetic 
differentiation of Arsenophonus in this or other spiders, investigations of more populations or species of spider need to be conducted. Bacterial symbionts can be transmitted vertically and horizontally, and these transmission modes allow symbionts to persist for long periods and rapidly spread in host populations as well as colonize novel hosts (Bright \& Bulgheresi, 2010). The widely distributed symbionts Wolbachia and Cardinium can be transferred both vertically and horizontally; they have extensive histories of movement both within and among species (Baldo et al., 2006; Raychoudhury et al., 2009; Russell et al., 2009; Oliver et al., 2010). Arsenophonus, as a clade of intracellular symbionts with a broad distribution, has also been found to be transmitted from parents to offspring or from one species to another. In the parasitoid Nasonia vitripennis, Arsenophonus can be transmitted to the next generation from parents (Huger et al., 1985; Gherna et al., 1991). In addition, in N. vitripennis, this symbiont can be transmitted to another species of parasitoid, $N$. giraulti (Duron et al., 2010). In the present study, we found that in the predator $P$. pseudoannulata, Arsenophonus could be maternally transmitted to the next generation, but the transmission rate was low (approximately 25\%). Moreover, we found the symbiont in the offspring of spiders that were not infected with Arsenophonus, at an infection rate of approximately $11 \%$, indicating that Arsenophonus in spiders has one or more transmission modes in addition to maternal transmission; possibilities include paternal transmission and spider parasitoid transmission. Although Arsenophonus has been detected in strawberry and beet crops (Zreik et al., 1998; Bressan et al., 2008), it has not been found in rice plants (Jiang et al., 2017). Therefore, we infer that Arsenophonus can be transmitted by parents of the spider, i.e., both maternally and paternally. Due to the difficulty of identifying the fathers of spiders in the field, we could not confirm a role of male spiders in Arsenophonus transmission in the present study. The possibility of male transmission needs to be verified in future work.

The symbiotic relationships between Arsenophonus and their hosts have attracted increasing interest. In $N$. vitripennis, a natural enemy of insect pests, Arsenophonus can induce death of male embryos (Gherna et al., 1991), and in rice planthoppers, this symbiont can improve host resistance to the entomopathogenic fungi Metarhizium anisopliae (Zhu et al., 2017). Arsenophonus has also been found or speculated to be involved in insect resistance (Pang et al., 2018). For example, it was found to decrease the resistance of rice brown planthoppers to the chemical insecticide imidachloprid (Liu \& Guo, 2019). A recent study found that Arsenophonus affected the amino acid requirements of aphids (Tian et al., 2019). However, to the best of our knowledge, the effects of Arsenophonus on spiders remain unknown. Thus, studies of Arsenophonus infection and transmission characteristics in spiders can help reveal the biological roles and evolution of this symbiont in the predators of insect pests. Studies of the interactions between Arsenophonus and their hosts are urgently needed.

\section{Conclusions}

Spider P. pseudoannulata populations are infected with the symbiont Arsenophonus at low rates. This symbiont can be vertically transmitted from female spiders to offspring but also may be transmitted by male spiders or other vectors. Arsenophonus populations are genetically differentiated among different geographical regions of spiders. 


\section{Declarations}

\section{Ethics approval and consent to participate}

The spiders used in our studies were collected from rice paddies in different locations in China, and no specific permissions were required for these locations. We confirm that these locations are not privatelyowned or protected in any way, and our field studies do not involve endangered or protected species.

\section{Consent to publish}

Not applicable.

\section{Availability of data and materials}

The datasets used and analysed during the current study are available from the corresponding author on reasonable request.

\section{Competing interests}

The authors declare that they have no competing interests.

\section{Funding}

This research was supported by grants from the National Natural Science Foundation of China $(31972265,31672027)$. The funding body had no role in the design of the experiment; the collection, analysis, and interpretation of the data; or the writing of the manuscript.

\section{Authors' contributions}

$\mathrm{HJ}$ and YD collected the samples from fields and analyzed the infection incidence of symbionts, DZ built the phylogenetic tree, $\mathrm{XL}$ designed the experiments and revised the manuscript, $\mathrm{HG}$ conceived and designed the experiments, and wrote the manuscript. All authors read and approved the final manuscript.

\section{References}

1. Pontes $\mathrm{MH}$, Dale C. Culture and manipulation of insect facultative symbionts. Trends Microbiol. 2006;14:406-12.

2. Duron O, Bouchon D, Boutin S, Bellamy L, Zhou L, Engelstädter J, Hurst GD. The diversity of reproductive parasites among arthropods: Wolbachia do not walk alone. BMC Biol. 2008;6:27.

3. Zug R, Hammerstein P. Bad guys turned nice? A critical assessment of Wolbachia mutualisms in arthropod hosts. Biol Rev. 2015;90:89-111.

4. Liu XD, Guo HF. Correlation between insecticide resistance and endosymboints Wolbachia and Rickettsia. Cur Opinion Insect Science 2019;33: 84-90. 
5. Nyffeler M, Benz G. Spiders in natural pest control: a review. J Appl Entomol. 1987;103:321-39.

6. Rowley SM, Raven RJ, McGraw EA. Wolbachia pipientis in Australian spiders. Curr Microbiol. 2004;49:208-14.

7. Goodacre SL, Martin OY, Thomas CF, G, Hewitt GM. Wolbachia and other endosymbiont infections in spiders. Mol Ecol. 2006;15:517-27.

8. Vanthournout B, Swaegers J, Hendrickx F. Spiders do not escape reproductive manipulations by Wolbachia. BMC Evol Biol. 2011;11:15.

9. Stefanini A, Duron O. Exploring the effect of the Cardinium endosymbiont on spiders. J Evol Biol. 2012;25:1521-30.

10. ZhangL, Yun Y, Hu G, Peng Y. Insights into the bacterial symbiont diversity in spiders. Ecol Evol. 2018;8:4899-906.

11. Gherna RL, Werren JH, WeisburgW, Cote R, Woese CR, Mandelco L, Brenner DJ. Arsenophonus nasoniae gen. nov., sp. Nov., the causative agent of the son-killer trait in the parasitic wasp Nasonia vitripennis. Internation J System Evol Microbiol. 1991;41:563-5.

12. Chiel E, Gottlieb Y, Zchori-Fein E, Mozes-Daube N, Katzir N, Inbar M, Ghanim M. Biotype-dependent secondary symbiont communities in sympatric populations of Bemisia tabaci. Bull Entomol Res. 2007;97:407-13.

13. Hansen AK, Jeong G, Paine TD, Stouthamer R. Frequency of secondary symbiont infection in an invasivepsyllid relates to parasitism pressure on a geographic scale in California. Appl Environ Microbiol. 2007;73:7531-35.

14. Nováková E, Hypša V, Moran NA. Arsenophonus, an emerging clade of intracellular symbionts with a broad host distribution. BMC Microbiol. 2009; 9,143.

15. Wagner SM, Martinez AJ, Ruan Y, Kim KL, Lenhart PA, Dehnel AC, Oliver KM, White JA. Facultative endosymbionts mediate dietary breadth in a polyphagous herbivore. Funct Ecol. 2015;29:1402-10.

16. Huger AM, Skinner SW, Werren JH. Bacterial infections associated with the son-killer trait in the parasitoid wasp, Nasonia (= Mormoniella) vitripennis (Hymenoptera, Pteromalidae). J Invertebr Pathol. 1985;46:272-80.

17. Duron $\mathrm{O}$, Wilkes TE, Hurst GDD. Interspecific transmission of a male-killing bacterium on an ecological timescale. Ecol Lett. 2010;13:1139-48.

18. Liu XD, Liu Y, Zhang XX, Liu TL, Zhu YL, Xu ZZ. Spider community and its coherency with Nilaparvata lugens in a single paddy-field. Acta Ecol Sin. 1999;19:876-81.

19. Li Y, Liu X, Guo H. Variations in endosymbiont infection between buprofezin-resistant and susceptible strains of Laodelphax striatellus (Fallén). Curr Microbiol. 2018. doi.org/10.1007/s00284-018-1436-X.

20. Thao ML, Clark MA, Baumann L, Brennan EB, Moran NA, Baumann P. Secondary endosymbionts of psyllids have been acquired multiple times. Curr Microbiol. 2000;41:300-4.

21. Nakamura Y, KawaiS, Yukuhiro F, Ito S, Gotoh T, Kisimoto R, Yanase T, Matsumoto Y, Kageyama D, Noda H. Prevalence of Cardinium bacteria in planthoppers and spider mites and taxonomic revision 
of "Candidatus Cardinium hertigii" based on detection of a new Cardinium group from biting midges. Appl Environ Microbiol. 2009;75:6757-63.

22. Vanbroekhoven K, Ryngaert A, Wattiau P, Mot R, Springael D. Acinetobacter diversity in environmental samples assessed by 16 S rRNA gene PCR-DGGE fingerprinting. FEMs Microbio Ecol. 2004;50:3750 .

23. Zhou WG, Rousset F, O'Neill S. Phylogeny and PCR-based classification of Wolbachia strains using wsp gene sequences. Proc R Soc Lond B. 1998;265:509-15.

24. Zhu H, Sun SJ, Dang HY. PCR detection of Serratia spp. using primers targeting $p f s$ and luxS genes involved in Al-2-dependent quorum sensing. Curr Microbiol. 2008;57:326-30.

25. Sanada-Morimura S, Matsumura M, Noda H. Male killing caused by a Spiroplasma symbiont in the small brown planthopper, Laodelphax striatellus. J Heredity. 2013;104:821-9.

26. Wang WX, Luo J, Lai FX, Fu Q. Identification and phylogenetic analysis of symbiotic bacteria Arsenophonus from the rice brown planthopper, Nilaparvata lugens (stål). Acta Entomol Sin. 2012;52:1002-10.

27. Qu LY, Lou YH, Fan HW, Ye YX, Huang HJ, Hu MQ, Zhu YN, Zhang CX. Two endosymbiotic bacteria, Wolbachia and Arsenophonus, in the brown planthopper Nilaparvata lugens. Symbiosis. 2013;61:47-53.

28. Jiang JJ, Wang FY, Huang LF, Chen HS, Yang L. Detection and analysis of four secondary endosymbiotic bacteria (Arsenophonus, Wolbachia, Spiroplasma and Cardinium) in the brown planthopper, Nilaparvata lugens (Homoptera: Delphaciade. Chinese J Appl Entomol. 2017;54:58393.

29. Vanthournout B, Hendrickx F. Endosymbiont dominated bacterial communities in a dwarf spider. PLoS One. 2015;10:e0117297. doi:10.1371/journal.pone.0117297.

30. Thao ML, Baumann P. Evidence for multiple acquisition of Arsenophonus by whitefly species (Sternorrhyncha: Aleyrodidae). Curr Microbiol. 2004;48:140-4.

31. Mouton L, Thierry M, Henri H, Baudin R, Gnankine O, Reynaud B, et al. Evidence of diversity and recombination in Arsenophonus symbionts of the Bemisia tabaci species complex. BMC Microbiol. 2012; 12 Suppl 1: S10. PMID: 22375811.

32. Bright M, Bulgheresi S. A complex journey: transmission of microbial symbionts. Nat Rev Microbiol. 2010;8:218-30.

33. Baldo L, Dunning Hotopp JC, Jolley KA, Bordenstein SR, Biber SA, Choudhury RR, Hayashi C, Maiden MCJ, Tettelin H, Werren JH. Multilocus sequence typing system for the endosymbiont Wolbachia pipientis. Appl Environ Microbiol. 2006;72:7098-110.

34. Raychoudhury R, Baldo L, Oliveira DC, Werren JH. Modes of acquisition of Wolbachia: horizontal transfer, hybrid introgression, and codivergence in the Nasonia species complex. Evolution. 2009;63:165-83.

35. Russell JA, Goldman-Huertas B, Moreau CS, Baldo L, Stahlhut JK, Werren JH, Pierce NE. Specialization and geographic isolation among Wolbachia symbionts from ants and lycaenid 
butterflies. Evolution. 2009;63:624-40.

36. Oliver KM, Degnan PH, Burke GR, Moran NA. Facultative symbionts in aphids and the horizontal transfer of ecologically important traits. Ann Rev Entomol. 2010;55:247-66.

37. Zreik L, Bore JM, Gamier M. Phylogenetic characterization of the bacterium-like organism associated with marginal chlorosis of strawberry and proposition of a Candidatus taxon for the organism, Candidatus Phlomobacter fragariae. Inter J Syst Evol Microbiol. 1998;48:257-61.

38. Bressan A, Semetey O, Nusillard B, Clair D, Boudon-Padieu E. Insect vectors (Hemiptera: Cixiidae) and pathogens associated with the disease syndrome "basses riehesses" of sugar beet in France. Plant Dis. 2008;92:113-9.

39. Zhu HH, Chen Y, Wan PJ, Wang WX, Lai FX, Fu Q. Influence of symbiotic bacteria Arsenophonus, rice variety and temperature on the incidence rate of Nilaparvata lugens to Metarhizium flavoviride. Chinese J Rice Science. 2017;31:643-51. (in Chinese).

40. Pang R, Chen M, Yue L, Xing K, Li T, Kang K, Liang Z, Yuan L, Zhang W. A distinct strain of Arsenophonus symbiont decrease insecticide resistance in its insect host. PLoS Genet. 2018;14:e1007725.

41. Tian PP, Chang CY, Miao NH, Li MY, Liu XD. Infections with Arsenophonus facultative endosymbionts alter performance of aphids (Aphis gossypii) on an amino-acid-deficient diet. Appl Environ Microbiol. 2019;85:e01407-19.

\section{Figures}

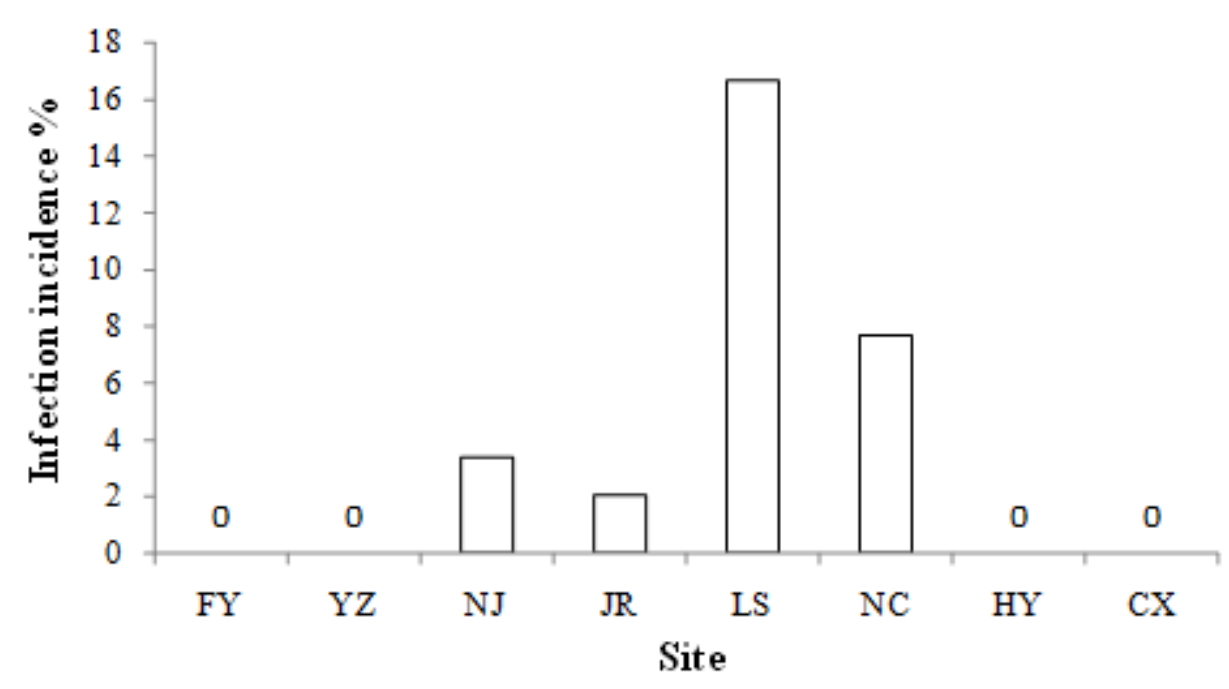

\section{Figure 1}

Incidence of infection with Arsenophonus in Pardosa pseudoannulata populations sampled at eight geographical sites in China. 

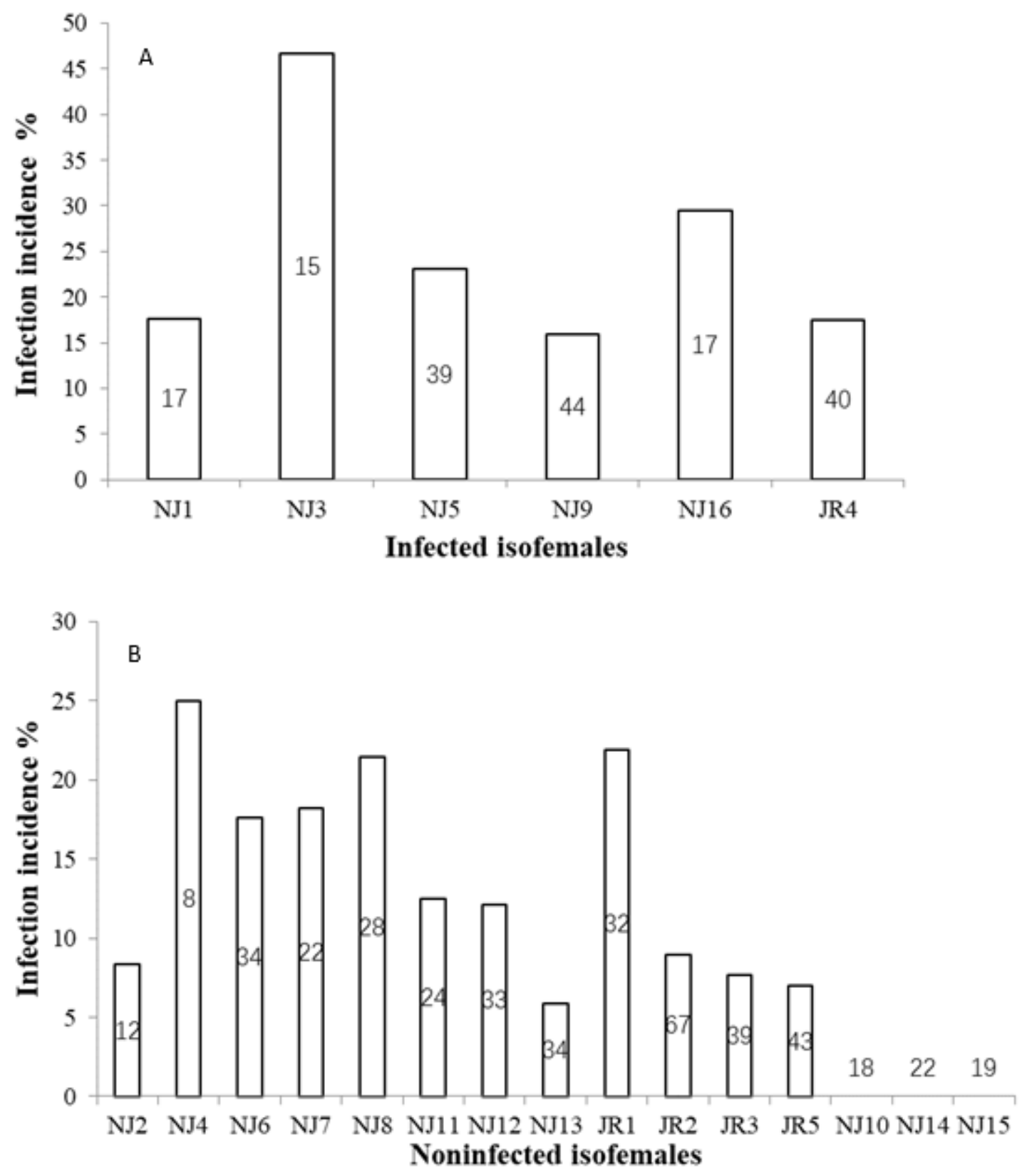

Figure 2

Maternal (A) and nonmaternal (B) transmission rates of Arsenophonus in 21 isofemales of Pardosa pseudoannulata. Numbers in bars represent the numbers of offspring of individual isofemales. 


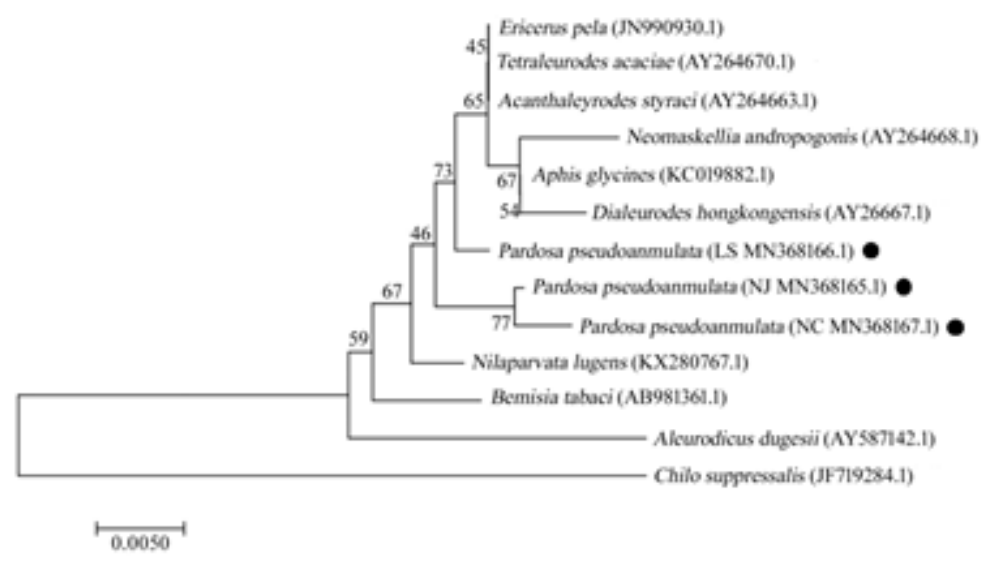

Figure 3

Phylogenetic tree based on the 23S rRNA gene sequences of Arsenophonus in Pardosa pseudoannulata and insect species using the neighbor-joining method. Numbers indicate bootstrap percentages at each node (1000 bootstraps). The nucleotide sequences of PCR-amplified fragments of Arsenophonus genes in our studies have been deposited in the GenBank nucleotide database under accession nos. MN368165 to MN368167. 